\title{
Evidence for increased thermogenesis in female C57BL/6J mice housed aboard the international space station
}

\author{
Carmen P. Wong ${ }^{1}$, Urszula T. Iwaniec ${ }^{1,2}$ and Russell T. Turner $\mathbb{I}^{1,2 凶}$
}

Sixteen-week-old female C57BL/6J mice were sacrificed aboard the International Space Station after 37 days of flight (RR-1 mission) and frozen carcasses returned to Earth. RNA was isolated from interscapular brown adipose tissue (BAT) and gonadal white adipose tissue (WAT). Spaceflight resulted in differential expression of genes in BAT consistent with increased non-shivering thermogenesis and differential expression of genes in WAT consistent with increased glucose uptake and metabolism, adipogenesis, and $\beta$-oxidation.

npj Microgravity (2021)7:23; https://doi.org/10.1038/s41526-021-00150-y

Mice are facultative daily heterotherms and, in contrast to humans who maintain a near constant core body temperature over a wide range of environmental temperatures, mice can experience dramatic transient reductions in core temperature (torpor) when exposed to temperatures below thermoneutral ${ }^{1,2}$. Because of their small mass, mice are much more dependent on shivering and non-shivering thermogenesis for maintaining core body temperature than larger animals ${ }^{3}$.

The recommended temperature for housing mice in a laboratory setting $\left(20-26^{\circ} \mathrm{C}\right)$ is well below their thermoneutral zone, which depending upon strain, sex and age typically ranges from $29-31^{\circ} \mathrm{C}^{4}$. Subthermoneutral housing induces adaptive responses in mice to increase generation of heat ${ }^{5}$. These adaptations include shivering thermogenesis, non-shivering thermogenesis, thermic effect of increased food consumption and physical activity. Sympathetic nervous system (SNS) neurotransmitters (norepinephrine and epinephrine) and the adipokine leptin are key factors involved in regulation of adaptive thermogenesis ${ }^{6,7}$. Subthermoneutral-housed mice use a variety of physiological and behavioral strategies to decrease their requirements for adaptive thermogenesis. These include entering torpor to lower core body temperature, and nest building, huddling and postural changes to minimize heat loss when exposed to a cool environment ${ }^{8,9}$.

The physiological demands required for successful adaptation to subthermoneutral housing by mice are considerable; for example, female mice housed at room temperature $\left(22^{\circ} \mathrm{C}\right)$ consumed $40 \%$ more food to achieve comparable weight gain and expressed 5-fold higher Ucp1 gene expression in BAT (nonshivering thermogenesis) compared to thermoneutral-housed $\left(32^{\circ} \mathrm{C}\right)$ mice $^{10}$. UCP-1 protein uncouples oxidative phosphorylation to produce heat instead of ATP. Extensive research supports the conclusion that by increasing UCP-1 protein levels, sympathetic signaling-driven non-shivering thermogenesis plays an important role in cold-induced thermoregulation in mice.

We hypothesize that spaceflight reduces the ability of mice to employ some of the strategies used to minimize adaptive thermogenesis, such as huddling and postural adjustments, resulting in an increased dependence on adaptive thermogenesis to maintain core body temperature. Group housing attenuates the increase in non-shivering thermogenesis in BAT in mice exposed to a cool environment ${ }^{11}$ but it is plausible that another aspect of the spaceflight environment increases sympathetic signaling, an important positive regulator of thermogenesis. Whatever the precise mechanism, increased thermogenesis is important because it influences multiple physiological processes ${ }^{12-15}$. Collateral changes associated with increased thermogenesis include cancellous bone loss, immune suppression, increases in glucocorticoid production, increases in blood pressure and heart rate, and altered tumor and tissue response to ionizing radiation ${ }^{16-18}$. Mechanistically, at least some of these responses are mediated by increased sympathetic outflow ${ }^{14,19,20}$.

Thus, activation of adaptive thermogenesis in mice housed in microgravity may introduce unrecognized and uncontrolled for confounding variables into spaceflight studies. We tested the hypothesis that nonshivering thermogenesis is increased in mice during spaceflight by measuring the effect of spaceflight on expression of genes related to energy metabolism in BAT and WAT in female C57BL/6J (B6) mice. In contrast to most prior spaceflight studies where flight animals were returned to Earth, the animals in this experiment (RR-1) were sacrificed aboard the International Space Station (ISS), avoiding the influence of restoration of normal gravitational loading.

Temperatures during the spaceflight mission ranged from a low of $21.3^{\circ} \mathrm{C}$ to a high of $28.0^{\circ} \mathrm{C}$. The average housing temperatures within the Habitats enclosing the mice aboard ISS were $26.0^{\circ} \mathrm{C}$ and $26.4{ }^{\circ} \mathrm{C}$ for flight and ground control animals, respectively. These housing temperatures, while above temperatures commonly used to house mice, are below thermoneutral for this species. Weight gain did not differ, but activity levels were higher in the flight animals, as was food and water depletion ${ }^{21}$, findings consistent with increased adaptive thermogenesis.

The effect of the spaceflight environment on differential expression of genes related to energy metabolism in BAT in flight animals is shown in Table 1. Transcript abundance of 13/84 genes were significantly altered in flight animals compared to ground controls. In particular, mice housed aboard ISS had 1.5x higher levels of Ucp-1 in BAT, providing direct evidence for elevated nonshivering thermogenesis. Several genes associated with adipogenesis and/or thermogenesis, including Adipoq, Ppargcla, Cdkn $1 a^{22}$, and $C f d$ were differentially expressed in BAT during spaceflight ${ }^{23}$. However, these changes may reflect adaptation to long duration

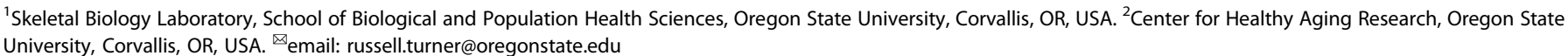


Table 1. Gene array showing fold changes for differentially expressed genes in interscapular brown adipose tissue (BAT) in mice sacrificed aboard the International Space Station after 37 days of flight (RR-1 mission) compared to ground controls.

Space flight vs. ground control

\begin{tabular}{llllll}
\hline Symbol & Fold change & $P<$ & Symbol & Fold change & $P<$ \\
\hline Acacb & -1.2 & 0.350 & Klf4 & -1.3 & 0.443
\end{tabular}

$\begin{array}{llllll}\text { Adig } & -1.1 & 0.599 & \text { Lep } & -1.1 & 0.603\end{array}$

Adipoq $\quad \mathbf{- 1 . 4}$

Adrb2 $\quad-1.1$

Agt $\quad-1$

Angpt2 $\quad-1.1$

$\operatorname{Axin}^{\mathrm{b}} \quad-1.2$

$B m p 2^{a} \quad-1.2$

Bmp4 ${ }^{b} \quad 1.2$

Bmp7 $\quad 1.1$

Ccnd $1 \quad-1$

Cdk4 -1.5

Cdkn1a $\quad 2.4$

Cdkn1b 1.1

Cebpa -1.1

Cebpb 1

Cebpd $\quad 1.2$

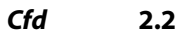

Creb1 $\quad-1.1$

Ddit3 $\quad 1.2$

$\operatorname{Dio2}^{\mathrm{b}} \quad-1.2$

Dkk1 1.4

Dlk1 1.2

E2f1 -1.5

Egr2 -1.6

Fabp4 -1

Fasn $\quad-1.1$

Fgf $^{b} \quad \mathbf{1 . 6}$

Fgf10 -1.3

Fgf2 $\quad \mathbf{1 . 5}$

Foxc2 -1.8

Foxo1 1

Gata2 -1.2

Gata3 -1.3

Hes $1 \quad 1.1$

Insr $\quad-1$

Irs1 $\quad-1.5$

Irs2 $\quad-2$

Jun $\quad-1.5$

KIf15 -1.1

KIf2 -1.2

KIf3

$-1.2$

aThis gene's average relative expression
reasonable in flight sample $(\mathrm{Ct}<30)$.

'This gene's relative expression level is low in both control and flight samples $(\mathrm{Ct}>30)$.
Table 2. Gene array showing fold changes for differentially expressed genes in gonadal white adipose tissue (WAT) in mice sacrificed aboard the International Space Station after 37 days of flight (RR-1 mission) compared to ground controls.

Space flight vs. ground contro

\begin{tabular}{|c|c|c|c|c|c|}
\hline Symbol & Fold change & $P<$ & Symbol & Fold change & $P<$ \\
\hline Acacb & 4 & 0.001 & Klf4 & 1.1 & 0.291 \\
\hline Adig & 1.4 & 0.014 & Lep & -1.4 & 0.662 \\
\hline Adipoq & -1.1 & 0.414 & Lipe & 1 & 0.932 \\
\hline Adrb2 $^{a}$ & 1.5 & 0.107 & Lmna & 1.3 & 0.042 \\
\hline Agt & 1.1 & 0.635 & $L p l$ & -1.2 & 0.145 \\
\hline Angpt2 & -1.5 & 0.102 & Lrp5 & 1.1 & 0.518 \\
\hline Axin1 & 1.5 & 0.117 & Mapk14 & 1.7 & 0.002 \\
\hline Bmp2 & 2.9 & 0.020 & Ncoa 2 & 1.3 & 0.102 \\
\hline$B m p 4^{a}$ & -1.2 & 0.945 & Ncor2 & 1.3 & 0.480 \\
\hline Bmp7 & 3.5 & 0.030 & NrOb2 & 2.4 & 0.048 \\
\hline Cend1 & 1.8 & 0.008 & Nr1h3 & 1.5 & 0.001 \\
\hline Cdk4 & 1.2 & 0.212 & $N r f 1$ & 2.1 & 0.046 \\
\hline Cdkn1a & 3 & 0.001 & Ppara $^{a}$ & 1.7 & 0.121 \\
\hline$C d k n 1 b$ & 1.3 & 0.104 & Ppard $^{a}$ & 2 & 0.003 \\
\hline Cebpa & 1 & 0.708 & Pparg & 1.3 & 0.031 \\
\hline Cebpb & 2.2 & 0.003 & Ppargcia & 2.1 & 0.098 \\
\hline Cebpd & 1.3 & 0.193 & Ppargc1b & 2.4 & 0.003 \\
\hline Cfd & 2.3 & 0.002 & Prdm16 & 2.2 & 0.063 \\
\hline Creb1 & 1.8 & 0.003 & $R b 1$ & 1.1 & 0.095 \\
\hline Ddit3 & 1.3 & 0.102 & Retn & 1.6 & 0.046 \\
\hline Dio2 & 5.5 & 0.042 & Runx1t1 ${ }^{a}$ & 3.2 & 0.009 \\
\hline$D k k 1^{b}$ & 3.5 & 0.108 & Rxra & 1.6 & 0.067 \\
\hline$D / k 1^{b}$ & 2.8 & 0.077 & Sfrp ${ }^{a}$ & -1 & 0.957 \\
\hline$E 2 f 1$ & 1.7 & 0.106 & Sfrp5 ${ }^{a}$ & 1.3 & 0.709 \\
\hline $\operatorname{Egr}^{b}$ & 1.8 & 0.194 & Shh & 5 & 0.014 \\
\hline Fabp4 & 1.2 & 0.094 & Sirt1 & 1.5 & 0.072 \\
\hline Fasn & 2.5 & 0.009 & Sirt2 & 1.2 & 0.049 \\
\hline Fgf1 & 1.3 & 0.840 & Sirt3 & 2 & 0.033 \\
\hline Fgf10 & 1.1 & 0.516 & Slc2a4 & 2.2 & 0.002 \\
\hline Fgf2 & -1.1 & 0.872 & Src ${ }^{a}$ & 2.2 & 0.018 \\
\hline Foxc2 ${ }^{b}$ & 2 & 0.102 & Srebf1 & 2.5 & $<0.001$ \\
\hline Foxo1 & 1.3 & 0.040 & $T a z$ & 1.5 & 0.051 \\
\hline Gata2 & 2.4 & 0.064 & Tcf7/2 & 1.6 & 0.007 \\
\hline Gata3 & 4.5 & 0.079 & $T s c 22 d 3$ & -1 & 0.726 \\
\hline $\operatorname{Hes} 1^{a}$ & 2.7 & 0.102 & Twist1 & 1.6 & 0.022 \\
\hline Insr & 1.3 & 0.089 & $U_{c p}{ }^{a}$ & 29.3 & 0.122 \\
\hline $\mid r s 1$ & 1 & 0.881 & $V d r^{b}$ & 3 & 0.145 \\
\hline Irs2 & -1.3 & 0.400 & $W n t 1^{b}$ & 2.9 & 0.080 \\
\hline Jun & -1.6 & 0.132 & Wnt10b ${ }^{b}$ & 4.1 & 0.210 \\
\hline KIf15 & -1.2 & 0.329 & $W n t 3 a^{b}$ & 4.2 & 0.075 \\
\hline Klf2 & -1.2 & 0.186 & Wnt5a & 1.9 & 0.052 \\
\hline KIf3 & 1.1 & 0.752 & $W n t 5 b^{b}$ & 2.2 & 0.112 \\
\hline
\end{tabular}

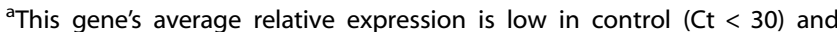
reasonable in flight sample $(\mathrm{Ct}<30)$.

${ }^{\text {b}}$ This gene's relative expression level is low in both control and flight samples $(\mathrm{Ct}>30)$ 
spaceflight. Regardless of the mechanisms for initiation, increased Ucp1-mediated thermogenesis in BAT may have had a major impact on adipose tissue turnover in WAT (Table 2). Transcript abundance of 30/84 genes were significantly altered in flight animals compared to ground controls. Notable changes, including higher expression levels for (1) Acacb (Acetyl-CoA carboxylase), a key gene in regulation of fatty acid oxidation, (2) Dio2 (Type II iodothyronine deiodinase), a key regulator of thyroid hormone action, (3) Sic2a4 (Glut 4), an insulin-regulated glucose transporter, and (4) Fasn (fatty acid synthase) which catalyzes the synthesis of palmitate, were observed in WAT of flight animals. There was also evidence for induction of Ucp-1; expression level for this gene was very low $(\mathrm{Ct}>30)$ in ground control mice and consistently detected in flight mice $(\mathrm{Ct}<30)$, suggesting browning of $\mathrm{WAT}^{24}$. We conclude from these findings that, in spite of comparable housing temperatures, adaptive thermogenesis is increased in BAT of mice housed aboard the ISS compared to ground controls. This is important because increased thermogenesis may exaggerate (e.g., bone loss) or alter (e.g., response to ionizing radiation) physiological responses to spaceflight in mice. Because of species specific differences in thermoregulation, this could impact the translatability of the animal studies to astronauts.

\section{METHODS}

\section{Spaceflight study}

Details of the spaceflight mission are published ${ }^{25}$. Animal protocol was reviewed and approved by the NASA Institutional Animal Care and Use Committee prior to the conduct of experiments. In brief, 16-week-old female B6 mice were sacrificed aboard the ISS after 37 days of flight and frozen carcasses were returned to Earth for tissue preparation and method validation as described ${ }^{21}$. Sensors in the Habitats (flight and ground) monitored and relayed information including component temperature and humidity. The data were logged at sampling rate of $1 \mathrm{~Hz}$. Ground control mice were sacrificed and processed using the same timelines and protocols as the flight animals.

\section{Gene expression study}

RNA was isolated from individual BAT ( $n=8$ /group) and WAT $(n=6 /$ group) samples. RNA integrity number (RIN) was assessed using an Agilent Bioanalyzer (Santa Clara, CA, USA). A RIN value of 5 and above is required to ensure reliable quantification of gene expression by RT-qPCR ${ }^{26,27}$. The RIN numbers (mean $\pm \mathrm{SE}, n=6$ /group) for RNA isolated from BAT from flight and ground control animals were $6.55 \pm 0.47$, and $7.42 \pm 0.46$, respectively. Thus RNA from both groups of animals were of good quality. mRNA was reverse transcribed into CDNA using SuperScript III First-Strand Synthesis SuperMix for qRT-PCR (ThermoFisher Scientific). Expression levels for genes related to adipogenesis was determined for BAT and WAT using the Mouse Adipogenesis RT ${ }^{2}$ Profiler PCR Array (Qiagen). Gene expression was normalized using GusB and ActB housekeeping genes, and relative quantification ( $\triangle \Delta \mathrm{Ct}$ method) was determined using $\mathrm{RT}^{2}$ Profiler PCR Array Data Analysis software (Qiagen).

\section{Reporting summary}

Further information on research design is available in the Nature Research Reporting Summary linked to this article.

\section{DATA AVAILABILITY}

Data are available in Supplemental Table 1 for BAT PCR array and Supplemental Table 2 for WAT PCR array.

Received: 18 November 2020; Accepted: 12 May 2021; Published online: 18 June 2021

\section{REFERENCES}

1. Swoap, S. J. \& Gutilla, M. J. Cardiovascular changes during daily torpor in the laboratory mouse. Am. J. Physiol. Regul. Integr. Comp. Physiol. 297, R769-774 (2009).

2. Brown, R. T. \& Baust, J. G. Time course of peripheral heterothermy in a homeotherm. Am. J. Physiol. 239, R126-129 (1980).

3. Tracy, C. R. Minimum size of mammalian homeotherms: role of the thermal environment. Science 198, 1034-1035 (1977).

4. Ganeshan, K. \& Chawla, A. Warming the mouse to model human diseases. Nat. Rev. Endocrinol. 13, 458-465 (2017).

5. Golozoubova, V. et al. Only UCP1 can mediate adaptive nonshivering thermogenesis in the cold. FASEB J. 15, 2048-2050 (2001).

6. Gavrilova, O. et al. Torpor in mice is induced by both leptin-dependent and -independent mechanisms. Proc. Natl Acad. Sci. USA 96, 14623-14628 (1999).

7. Swoap, S. J. \& Weinshenker, D. Norepinephrine controls both torpor initiation and emergence via distinct mechanisms in the mouse. PLOS ONE 3, e4038 (2008).

8. Barnett, S. A. Adaptation of mice to cold. Biol. Rev. Camb. Philos. Soc. 40, 5-51 (1965).

9. Hankenson, F. C., Marx, J. O., Gordon, C. J. \& David, J. M. Effects of rodent thermoregulation on animal models in the research environment. Comp. Med. 68, 425-438 (2018).

10. Iwaniec, U. T. et al. Room temperature housing results in premature cancellous bone loss in growing female mice: implications for the mouse as a preclinical model for age-related bone loss. Osteoporos. Int. 27, 3091-3101 (2016).

11. Maher, R. L., Barbash, S. M., Lynch, D. V. \& Swoap, S. J. Group housing and nest building only slightly ameliorate the cold stress of typical housing in female C57BL/6J mice. Am. J. Physiol. Regul. Integr. Comp. Physiol. 308, R1070-1079 (2015).

12. Clapham, J. C. Central control of thermogenesis. Neuropharmacology 63, 111-123 (2012).

13. Gordon, C. J. The mouse thermoregulatory system: Its impact on translating biomedical data to humans. Physiol. Behav. 179, 55-66 (2017).

14. Turner, R. T. et al. Effects of propranolol on bone, white adipose tissue, and bone marrow adipose tissue in mice housed at room temperature or thermoneutral temperature. Front. Endocrinol. 11, 117 (2020).

15. Yang, X. \& Ruan, H. B. Neuronal control of adaptive thermogenesis. Front. Endocrinol. 6, 149 (2015).

16. Fischer, A. W., Cannon, B. \& Nedergaard, J. Optimal housing temperatures for mice to mimic the thermal environment of humans: an experimental study. Mol. Metab. 7, 161-170 (2018).

17. Hylander, B. L. \& Repasky, E. A. Thermoneutrality, mice, and cancer: a heated opinion. Trends Cancer 2, 166-175 (2016).

18. Martin, S. A. et al. Thermoneutral housing attenuates premature cancellous bone loss in male C57BL/6J mice. Endocr. Connect 8, 1455-1467 (2019).

19. Hylander, B. L., Eng, J. W. \& Repasky, E. A. The impact of housing temperatureinduced chronic stress on preclinical mouse tumor models and therapeutic responses: An important role for the nervous system. Adv. Exp. Med Biol. 1036, 173-189 (2017).

20. Povinelli, B. J. et al. Standard sub-thermoneutral caging temperature influences radiosensitivity of hematopoietic stem and progenitor cells. PLOS ONE 10, e0120078 (2015).

21. Choi, S. Y. et al. Validation of a new rodent experimental system to investigate consequences of long duration space habitation. Sci. Rep. 10, 2336 (2020).

22. Krstic, J., Reinisch, I., Schupp, M., Schulz, T. J. \& Prokesch, A. p53 functions in adipose tissue metabolism and homeostasis. Int. J. Mol. Sci. 19, 2622 (2018).

23. Qiao, L. et al. Adiponectin reduces thermogenesis by inhibiting brown adipose tissue activation in mice. Diabetologia 57, 1027-1036 (2014).

24. Collins, S. beta-Adrenoceptor signaling networks in adipocytes for recruiting stored fat and energy expenditure. Front Endocrinol. (Lausanne) 2, 102 (2011).

25. Ronca, A. E. et al. Behavior of mice aboard the International Space Station. Sci. Rep. 9, 4717 (2019).

26. Padhi, B. K., Singh, M., Rosales, M., Pelletier, G. \& Cakmak, S. A PCR-based quantitative assay for the evaluation of mRNA integrity in rat samples. Biomol. Detect Quantif. 15, 18-23 (2018).

27. Fleige, S. \& Pfaffl, M. W. RNA integrity and the effect on the real-time qRT-PCR performance. Mol. Asp. Med 27, 126-139 (2006).

\section{ACKNOWLEDGEMENTS}

This work was supported by NASA (80NSSC19K0430 and NNX15AL15G). 


\section{AUTHOR CONTRIBUTIONS}

C.P.W.: conceptualization, investigation, analysis, writing-review \& editing. U.T.I.: conceptualization, writing-review \& editing. R.T.T.: conceptualization, funding acquisition, writing - original draft preparation, review \& editing.

\section{COMPETING INTERESTS}

The authors declare no competing interests.

\section{ADDITIONAL INFORMATION}

Supplementary information The online version contains supplementary material available at https://doi.org/10.1038/s41526-021-00150-y.

Correspondence and requests for materials should be addressed to R.T.T.

Reprints and permission information is available at http://www.nature.com/ reprints
Publisher's note Springer Nature remains neutral with regard to jurisdictional claims in published maps and institutional affiliations.

(i) Open Access This article is licensed under a Creative Commons adaptation, distribution and reproduction in any medium or format, as long as you give appropriate credit to the original author(s) and the source, provide a link to the Creative Commons license, and indicate if changes were made. The images or other third party material in this article are included in the article's Creative Commons license, unless indicated otherwise in a credit line to the material. If material is not included in the article's Creative Commons license and your intended use is not permitted by statutory regulation or exceeds the permitted use, you will need to obtain permission directly from the copyright holder. To view a copy of this license, visit http://creativecommons. org/licenses/by/4.0/.

(c) The Author(s) 2021 